\title{
Directional Feedback Delay Network
}

\author{
BENOIT ALARY ${ }^{1}$, ARCHONTIS POLITIS ${ }^{1,2}$, SEBASTIAN J. SCHLECHT ${ }^{1,3}$, AND \\ (Benoit.Alary@ Aalto.fi)
}

\author{
VESA VÄLIMÄKI, ${ }^{1}$ AES Fellow
}

\author{
${ }^{1}$ Acoustics Lab, Dept. of Signal Processing and Acoustics, Aalto University, Espoo, Finland \\ ${ }^{2}$ Faculty of Information Technology and Communication Sciences, Tampere University, Tampere, Finland \\ ${ }^{3}$ International Audio Laboratories Erlangen*, Erlangen, Germany
}

\begin{abstract}
Artificial reverberation algorithms are used to enhance dry audio signals. Delay-based reverberators can produce a realistic effect at a reasonable computational cost. While the recent popularity of spatial audio algorithms is mainly related to the reproduction of the perceived direction of sound sources, there is also a need to spatialize the reverberant sound field. Usually multichannel reverberation algorithms output a series of decorrelated signals yielding an isotropic energy decay. This means that the reverberation time is uniform in all directions. However, the acoustics of physical spaces can exhibit more complex direction-dependent characteristics. This paper proposes a new method to control the directional distribution of energy over time, within a delay-based reverberator, capable of producing a directional impulse response with anisotropic energy decay. We present a method using multichannel delay lines in conjunction with a direction-dependent transform in the spherical harmonic domain to control the direction-dependent decay of the late reverberation. The new reverberator extends the feedback delay network, retaining its time-frequency domain characteristics. The proposed directional feedback delay network reverberator can produce non-uniform direction-dependent decay time, suitable for anisotropic decay reproduction on a loudspeaker array or in binaural playback through the use of ambisonics.
\end{abstract}

\section{INTRODUCTION}

Delay-based digital artificial reverberation algorithms have been prominently used for over 50 years $[1,2]$. They were originally developed to use delay lines as an efficient way to simulate the buildup of echoes in a concert hall. Although more accurate reproduction methods have been developed since, these techniques are still widely popular due to their desirable aesthetic properties and computational efficiency [2]. By recirculating signals through multiple delay lines, the echo density can buildup [3] while gains and filters placed within the recirculation paths can be used to control the frequency-dependent decay property of the reverberator $[4,5]$.

The Feedback Delay Network (FDN) is a popular method that generalizes the coupling of multiple recirculating delay lines to form a reverberation algorithm [4, 2]. Gerzon [6, 7] first proposed a unitary matrix to control the recirculation in a network of allpass filters, whereas Stautner and Puckette

${ }^{*}$ The International Audio Laboratories Erlangen are a joint institution of the Friedrich-Alexander-Universität ErlangenNürnberg (FAU) and Fraunhofer Institut für Integrierte Schaltungen IIS.
[8] generalized the use of delay networks to design multichannel reverberators. The formal design of a tunable FDN was presented by Jot and Chaigne [4]. They studied the use of absorptive filters in these systems to maximize the mode density of the response while controlling the decay rate of different frequencies and ensuring consistency within frequency bands. The central lossless property of FDNs has been extensively studied by Rocchesso and Smith [9] and by Schlecht and Habets [10].

For multichannel sound reproduction Gerzon [6], as well as Stautner and Puckette [8], presented a method that distributes the decorrelated output of different delay lines to a set of spatially arranged loudspeakers. In [11], De Sena et al. introduced a physically informed recirculating delay system, using digital waveguide networks, capable of positioning the output at the location of the first-order early reflections in a virtual shoebox. This design was further expanded to include physically informed second-order reflections [12]. In [13] the recirculating matrix of an FDN was used to alter the output positioning and is capable of reproducing scenarios such as decoupled rooms.

In many applications of multichannel sound reproduction, the energy decay is assumed to be isotropic, emphasizing the directionality of the direct sound [14]. A sound 
field is isotropic when its mean energy distribution is uniform in all directions at a given time. In a reverberant sound field, the energy decay is isotropic when the reverberation time of the sound incident to a listener is the same in all directions. Therefore, we make a distinction between an isotropic sound field, considering the mean energy at all directions, and an isotropic energy decay, considering the mean reverberation time at all directions. The former is a property of the signal, whereas the latter is a property of a spatial impulse response (SIR). A truly isotropic energy decay can only be produced in a carefully designed reverberation chamber, but normal listening environments often exhibit at least slightly anisotropic reverberation properties. This can be due to the shape, the absorption properties of various materials, or the location of a listener in a space [1519]. While the perceptual threshold of anisotropic energy decay remains to be established, recent studies demonstrate that a spatially anisotropic sound field can be perceivable [20].

One existing reproduction method for anisotropic energy decay is through the use of ambisonic impulse responses. Ambisonics is based on spherical representations of a sound field, originally developed in the 1970s and has recently regained popularity due to its practical use as a spatial audio format for virtual reality [21]. Capturing a SIR using an ambisonic microphone and convolving it with a dry signal is a common ambisonic reverberation method [22]. This method can inherently capture and reproduce the spatial properties of the sound field, including anisotropic late reverberation [23, 24]. Virtual acoustics, such as raybased methods [25], can also offer the means to reproduce anisotropic energy decay, provided that the direction of arrival of each individually computed reflection is encoded into the output. Nonetheless, delay-based reverberation algorithms remain popular by offering a flexible parametric way to set the reverberation time of the system while remaining computationally efficient.

Recently, several designs for an ambisonic reverberator have been proposed. In [26], the multichannel reverberation algorithm introduced in [8] was expanded by converting from a first-order ambisonic signal, the B-Format, to a microphone array format, the A-Format, for delay processing. This method was further developed in [27] to control the energy weighting in different output directions independently. By using directional gain controls on the output, these approaches can produce an anisotropic sound field. However, since the direction-dependent energy decay rate will remain the same for all directions, the energy decay will remain isotropic.

This paper proposes a novel method to control the energy decay of a reverberant sound field within a delaybased artificial reverberation algorithm. Through the use of multichannel delay lines, we can preserve and alter the direction-dependent distribution of the delayed signals. By making use of these multichannel delay lines, a directiondependent weighting function can iteratively modify the recirculating signals to create direction-dependent reverberation times. By analyzing an existing space to calibrate the reverberation times in the system, the proposed re- verberator is capable of recreating a measured anisotropic energy decay.

Sec. 1 of this paper gives background information on the FDN, direction-dependent reverberation, and spherical harmonic domain processing. Sec. 2 introduces the Directional Feedback Delay Network (DFDN) method and its different components. Sec. 3 details the directional weighting transform used in the system, and Sec. 4 demonstrates a specific case study. Sec. 5 concludes the paper.

\section{BACKGROUND}

\subsection{Feedback Delay Network}

An FDN consists of a set of delay lines interconnected through a feedback matrix that defines the recirculating gain of each connection $[4,8]$, as shown in Fig. 1. The matrix is typically chosen to be orthogonal to create a lossless prototype for the FDN before any attenuation is introduced in the system. The FDN can be described by the difference equations $[4,9]$

$$
\begin{aligned}
y(n) & =\sum_{i=1}^{N} c_{i} g_{i} s_{i}(n)+d x(n), \\
s_{i}\left(n+m_{i}\right) & =\sum_{j=1}^{N} A_{i j} g_{j} s_{j}(n)+b_{i} x(n),
\end{aligned}
$$

where $y(n)$ is the sum of the input $x(n)$ and the recursive summed output $s_{i}(n)$ of the $i$ th delay line of length $m_{i}$, weighted by the input and output coefficients $b_{i}$ and $c_{i}$, respectively. The direct-path gain coefficient is $d$, and $g_{i}$ is the gain coefficient of a given delay line. Upon recirculation, the state values $s_{i}(n)$ are attenuated by the matrix $A_{i j}$ (see Fig. 1).

To control the decay of the system, gain factors $g_{i}$ are applied at the output of each delay line. A common parameter of a reverberator is the reverberation time $T_{60}$, defined as the amount of time required for the impulse-response energy to decay by $60 \mathrm{~dB}$ [28]. To parametrize the attenuation of an FDN, we use a target $T_{60}$ to obtain a per-sample attenuation value for a given sample rate $f_{\mathrm{s}}$ through

$$
g_{\mathrm{dB}}=-60 \frac{1}{T_{60} f_{\mathrm{s}}},
$$

which is converted from the logarithmic scale to the linear scale using

$$
g_{\text {lin }}=10^{\frac{g_{\mathrm{dB}}}{20}}
$$

in order to obtain the target attenuation at the output of each delay line from

$$
g_{i}=\left(g_{\text {lin }}\right)^{m_{i}} .
$$

This process can be extended to multiple, frequencydependent, $T_{60}$ values to parametrize absorptive filters $[4,5]$.

\subsection{Anisotropic Energy Decay}

To describe the behavior of an anisotropic energy decay within a reverberator, the reverberation time can be 


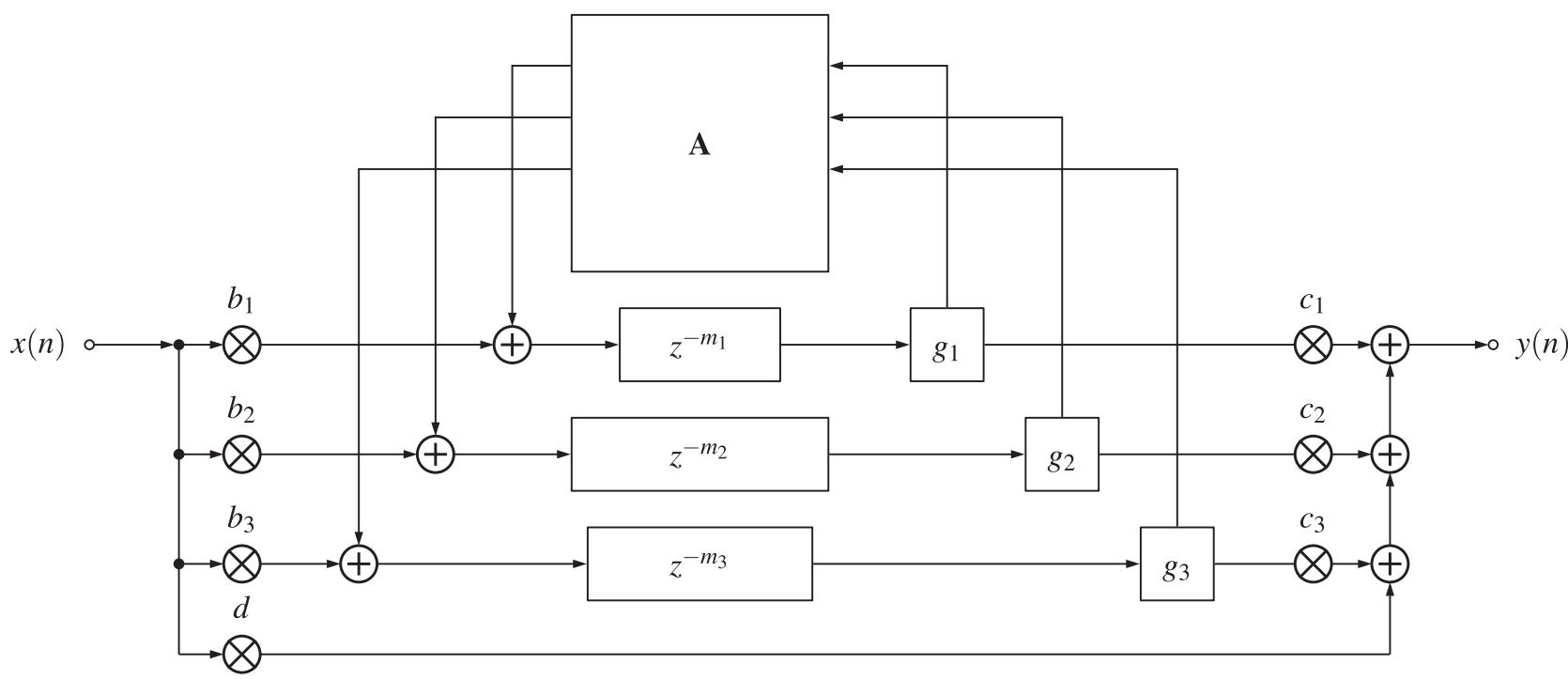

Fig. 1. Flow diagram of a conventional FDN.

specified as a direction-dependent parameter $T_{60}(\phi, \theta)$ for a given azimuth $\phi$ and elevation $\theta$. Furthermore, considering a maximum reverberation time $T_{60}^{\max }=\max \left\{T_{60}(\phi, \theta)\right\}$ along with a direction-dependent deviation $\Delta T_{60}(\phi, \theta)$ to characterize the deviation at a given direction is beneficial. These parameters should satisfy the following relationship:

$$
T_{60}(\phi, \theta)=T_{60}^{\max }-\Delta T_{60}(\phi, \theta) .
$$

\subsection{Ambisonics}

Ambisonics is a spatial audio format with a uniform spatial resolution in all directions, suitable for encoding arbitrary sound scenes and for spatial sound reproduction in arbitrary setups [21, 29-31]. It is based on spatial basis functions, the spherical harmonics (SHs), while the processing of the ambisonic signals occurs in the spherical harmonic domain (SHD). More specifically, when sound sources are in the far-field, an arbitrary sound field can be described as a continuous distribution of plane waves with amplitudes $s(n, \phi, \theta)$, each incident from azimuth $\phi$ and elevation $\theta$. The ambisonic signal vector $\mathbf{s}(n)=\left[s_{1}(n), \ldots, s_{Q}(n)\right]^{\mathrm{T}}$, up to a spatial resolution determined by the ambisonic order $L$, is given by the spherical harmonic transform (SHT) of the amplitude density

$$
\begin{aligned}
\mathbf{s}(n) & =\operatorname{SHT}[s(n, \phi, \theta)] \\
& =\int_{\phi=0}^{2 \pi} \int_{\theta=-\pi / 2}^{\pi / 2} s(n, \phi, \theta) \mathbf{y}(\phi, \theta) \cos \theta \mathrm{d} \theta \mathrm{d} \phi,
\end{aligned}
$$

where $\mathbf{y}(\phi, \theta)$ contains all $Q=(L+1)^{2}$ spherical harmonics up to order $L$. In practice, a discrete SHT (DSHT) can be realized as

$$
\mathbf{s}(n)=\frac{4 \pi}{K} \mathbf{Y}_{\text {uni }}^{\mathrm{T}} \mathbf{s}(n)
$$

where $\mathbf{Y}_{\text {uni }}=\left[\mathbf{y}\left(\phi_{1}, \theta_{1}\right), \ldots, \mathbf{y}\left(\phi_{K}, \theta_{K}\right)\right]^{\mathrm{T}}$ is a matrix of SHs sampled at $K \geq(L+1)^{2}$ points of a uniform spherical point set, suitable for the SHT without sampling error up to order $L$, such as a spherical $t$-design of degree $t=2 L$
[32]. Moreover, $\mathbf{s}(n)=\left[s\left(n, \phi_{1}, \theta_{1}\right), \ldots, s\left(n, \phi_{K}, \theta_{K}\right)\right]^{\mathrm{T}}$ is the sampled amplitude distribution at the same points.

The inverse SHT is performed through the expansion

$$
\tilde{s}(n, \phi, \theta)=\operatorname{ISHT}[\mathbf{s}(n)]=\mathbf{y}^{\mathrm{T}}(\phi, \theta) \mathbf{s}(n),
$$

which also constitutes a plane-wave decomposition of the sound scene. If the original distribution is spatially bandlimited to the order $L$ of the transform, then $\tilde{s}(n, \phi, \theta)=$ $s(n, \phi, \theta)$. Encoding a plane-wave source carrying a signal $t(n)$, incident from $\left(\phi_{0}, \theta_{0}\right)$ in the SHD, is realized as

$$
\mathbf{s}(n)=t(n) \mathbf{y}\left(\phi_{0}, \theta_{0}\right) \text {. }
$$

We use orthonormalized real SHs, common in higherorder Ambisonics (N3D ambisonic convention [31]), with the $A C N$ ordering of the ambisonic channels, according to which

$$
\begin{aligned}
{[\mathbf{y}(\phi, \theta)]_{q} } & =Y_{l d}(\phi, \theta), & \text { with } q & =1,2, \ldots,(L+1)^{2} \\
{[\mathbf{s}(n)]_{q} } & =s_{l d}(n), & \text { and } q & =l^{2}+l+d+1,
\end{aligned}
$$

with $l$ and $d$ being the spherical harmonic order and degree, respectively.

\subsection{Spatial Transformations}

Previous publications on Ambisonics covered fundamental spatial transformations that can be applied in the SHD, such as rotation and mirroring of a sound scene [29-31]. Such operations do not modify the direction-dependent energy distribution itself, only its orientation in space.

More complex transformations, which can apply a predefined directional weighting to the sound scene or warp directionally certain regions towards other directions, have also been demonstrated, initially for first-order Ambisonics $[33,29]$ and then extended to Ambisonics of any order [34-36].

However, such transformations are neither energy preserving nor order preserving, meaning that higher orders are required to capture the effect of the transform than the 


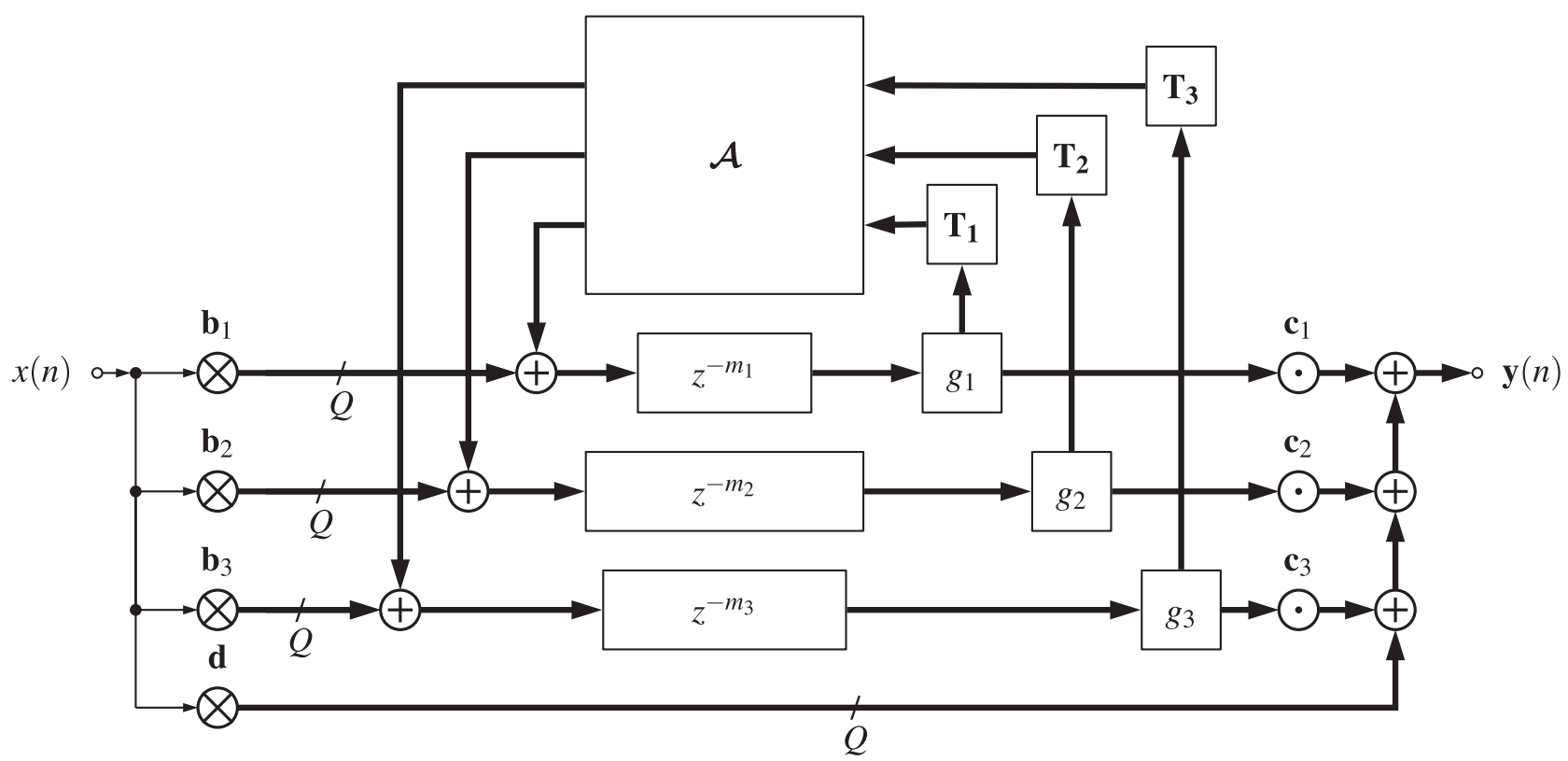

Fig. 2. DFDN flow diagram. The thick lines represent multiple connections associated with a delay-line group containing $Q$ delay lines.

order of the input signals. The directional reverberator design proposed here makes use of a directional weighting function, which we detail below.

Considering that we want to modify the original sound distribution $s(n, \phi, \theta)$ with a real directional weighting $g(\phi$, $\theta)$, we obtain a modified signal distribution

$$
s^{\prime}(n, \phi, \theta)=s(n, \phi, \theta) g(\phi, \theta) .
$$

If the original ambisonic scene is of order $L$ and the weighting function is of order $L^{\prime}$, then the resulting modified scene is of order $L+L^{\prime}$. This property follows from the fact that the basis functions, the SHs, constitute trigonometric polynomials, and the directional weighting function relates to a polynomial multiplication in which the product of two polynomials is a polynomial of degree equal to the sum of the two degrees. This operation can be expressed conveniently directly on the ambisonic signals through a linear transformation matrix $\mathbf{T}$. By expanding $s(n, \phi, \theta)$ and $g(\phi, \theta)$ using the inverse SHT, and taking the SHT of their product, we end up with the relation

$$
\mathbf{s}^{\prime}(n)=\mathbf{T} \mathbf{s}(n),
$$

where $\mathbf{T}$ is the $\left(L+L^{\prime}+1\right)^{2} \times(L+1)^{2}$ matrix operator transforming the initial distribution to the modified one. Details on the construction of $\mathbf{T}$ with respect to the directional weighting function $g(\phi, \theta)$ are presented in Sec. 4.

\section{DIRECTIONAL FEEDBACK DELAY NETWORK}

The DFDN is an extension to a conventional FDN capable of producing direction-dependent reverberation times. Individual delay lines are expanded into a set of multichannel signals by encoding a mono input signal in the SH domain, as in Eq. (11). A directional energy weighting function is included in the recirculating path to modify the direction-dependent energy decay $T_{60}(\phi, \theta)$, which can be tuned to resemble the anisotropic energy decay behavior of an existing space. Fig. 2 shows the structure of the DFDN reverberator. The signal $x(n)$ is the discrete-time input signal encoded into an ambisonic signal through $Q$ channels using the input gain vector $b_{i}$, which may be used to give the input signal an incident direction. Delay lines within a delay-line group have the same delay length $m_{i}$. The delay-line groups are attenuated by a common gain factor $g_{i}$, which should account for the smallest attenuation required by the system. The individual $g_{i}$ in Eq. (5) is obtained using $T_{60}^{\max }$ instead of $T_{60}$ in Eq. (3) along with the respective delay length $m_{i}$, starting with

$$
g_{\mathrm{dB}}=-60 \frac{1}{T_{60}^{\max f_{\mathrm{s}}}} .
$$

Each matrix $\mathbf{T}_{i}$ is the directional weighting matrix transform, that takes a set of ambisonic signals as the input and outputs signals in the same format. $\mathcal{A}$ is the orthogonal recirculation matrix that defines the recirculation gain between the delay lines.

\subsection{Input}

The directional weighting function is designed to control the direction-dependent decay of the late reverberation. For this reason, processing and positioning direct sound and the early reflections independently by sending them through a distinct parallel signal path is preferable. Nonetheless, the desired initial energy distribution should be used to encode the input signal into Ambisonics. Different methods can be used based on the desired perceptual effect. In our experiments, we chose to encode the mono signals into an omnidirectional incident sound field to highlight the directional attributes of the late reverberation. Therefore, for an omnidirectional source, the input gain of the direct sound of the $i$ th SH delay line is

$\mathbf{b}_{i}=[1,0,0, \ldots 0]$, 


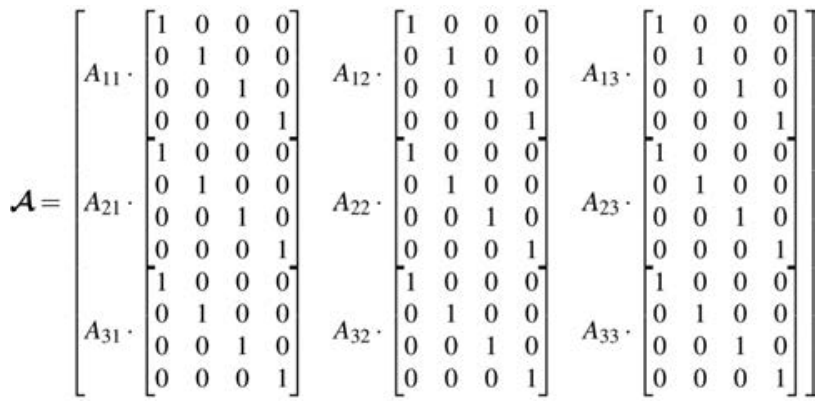

Fig. 3. Creation of the new block matrix through the Kronecker product.

where $\mathbf{b}_{i}$ is a $Q \times 1$ vector. The gain $\mathbf{d}$ can be set via the physically motivated incident direction of the direct sound using Eq. (11).

\subsection{Multichannel Delay Lines}

The difference equation of the $i$ th delay line of a regular FDN (see Eqs. (1) and (2)) is

$$
s_{i}(n)=\tilde{s}_{i}\left(n-m_{i}\right),
$$

where $\tilde{s}_{i}(n)$ is the input of the $i$ th delay line. The output of each delay line $s_{i}(n)$ in a regular FDN corresponds to the output of a delay-line group $\mathbf{s}_{i}(n)$ in the DFDN containing $Q$ sub-delay lines, one for each $\mathrm{SH}$ channel, all sharing the same delay length $m_{i}$. Thus, $\mathbf{s}_{i}(n)$ is a $Q \times 1$ vector with the same ordering as in Eq. (12). The difference equation of the SH delay line is then

$$
\mathbf{s}_{i}(n)=\tilde{\mathbf{s}}_{i}\left(n-m_{i}\right) \text {. }
$$

\subsection{Multichannel Recirculating Matrix}

To build the lossless prototype, the recirculating matrix of an FDN, which defines the recirculation gain from every delay line, is constrained to be orthogonal. In the DFDN, the recirculation of a given channel is limited to its corresponding channel within each delay-line group, which allows to preserve cohesion within the ambisonic channels. This means that the gains in the recirculation matrix should be set to zero for all the other channels. For this purpose, the new recirculating matrix $\mathcal{A}$ is created using the Kronecker product $(\otimes)$ between an existing FDN matrix A and the identity matrix $\mathbf{I}$ of size corresponding to the number of channels $Q$, expressed as

$$
\mathcal{A}=\mathbf{A} \otimes \mathbf{I}_{Q},
$$

which can be expanded into a $12 \times 12$ matrix for a fourchannel first-order ambisonic configuration with three delay lines (Fig. 3). The Kronecker product retains orthogonality when the two matrices involved in the operation are orthogonal themselves [37, 38].

\subsection{Recursive Spatial Transform}

As stated previously, the attenuation of an FDN is designed to make use of the recursive nature of the algorithm by using gain values or absorptive filters calculated from the length of each delay line. For the DFDN, we propose a spatial transform, represented by $\mathbf{T}_{i}$ in Fig. 2, that can recursively alter the energy in different directions to alter the reverberation times. The transform operates on ambisonic signals, and therefore, a delay-line group containing a set of ambisonic channels is used as the input. The transform is a matrix which is multiplied by the ambisonic signal of each delay-line group. While the transform aims to alter the direction-dependent decay, it must not introduce more energy into the system to ensure stability. Full details on the proposed transform are given in the next section. The complete transform matrix for the delay network can be constructed with a block diagonal matrix $\mathcal{T}$ :

$$
\mathcal{T}=\left[\begin{array}{ccc}
\mathbf{T}_{1} & \mathbf{0} & \mathbf{0} \\
\mathbf{0} & \mathbf{T}_{2} & \mathbf{0} \\
\mathbf{0} & \mathbf{0} & \mathbf{T}_{3}
\end{array}\right]
$$

Since acoustic phenomena are frequency-dependent, having a system behaving with frequency-dependent characteristics may be desirable as well. Frequency and direction-dependent features can be obtained by first splitting the output of each recirculating delay-line group using a filter bank and then connecting each filter output to a different directional weighting transform to obtain the frequency-dependent characteristics. The signals are then mixed back together before entering the recirculating matrix.

\subsection{Output}

Finally, each delay-line group is attenuated by vector $\mathbf{c}_{i}$, which allows direction-dependent gain control of the final output, and each delay-line group is mixed together with the direct sound to produce the final output $\mathbf{y}$. Since the output signal is already in the SHD, it can be decoded spatially to a loudspeaker array or binaurally to headphones using the appropriate decoding matrix [31]. The complete formula of the DFDN is given by modifying Eq. (2) as follows:

$$
\mathbf{y}(n)=\sum_{i=1}^{N} g_{i} \mathbf{c}_{i} \odot \mathbf{s}_{i}(n)+\mathbf{d} x(n),
$$

where $\odot$ denotes element-wise multiplication (Hadamard product) and

$$
\mathbf{s}_{i}\left(n+m_{i}\right)=\sum_{j=1}^{N} A_{i j} g_{j} \mathbf{T}_{j} \mathbf{s}_{j}(n)+\mathbf{b}_{i} x(n) .
$$

\section{DIRECTIONAL WEIGHTING TRANSFORM}

The directional weighting matrix (DWM) transform is a matrix operator that modifies the direction-dependent energy distribution of an ambisonic signal. By adding this operator in the recirculation path of the reverberator, we can control how the energy of the late reverberation is gradually distributed spatially. To construct the transform, we first specify directional decay times, which are converted into a directional weighting function. The transform itself takes the form of a matrix multiplied by the multichannel signals of each ambisonic delay-line group as expressed in Eq. (15). However, the full DWM transform generates 
new higher-order signals that require more output channels than input channels. Therefore, the transform matrix is truncated to retain the original SH order. The following section explains the DWM construction.

\subsection{Determining Directional Gain}

To define the DWM operator, a direction-dependent $g_{i}(\phi$, $\theta)$ must be calculated to yield a $\Delta T_{60}(\phi, \theta)$ for each delay line. Since a global attenuation $g_{i}$ based on $T_{60}^{\max }$ is already part of the system, it needs to subtracted. The per-sample direction-dependent gain factor $g_{i}(\phi, \theta)$ is obtained by modifying Eq. (3) to (5) as follows:

$$
\begin{aligned}
g_{\mathrm{dB}}(\phi, \theta) & =\frac{-60}{f_{\mathrm{s}}}\left(\frac{1}{T_{60}(\phi, \theta)}-\frac{1}{T_{60}^{\max }}\right), \\
& =\frac{-60}{f_{\mathrm{s}}}\left(\frac{\Delta T_{60}(\phi, \theta)}{T_{60}(\phi, \theta) T_{60}^{\max }}\right), \\
g_{\operatorname{lin}}(\phi, \theta) & =10^{\frac{g_{\mathrm{dB}}(\phi, \theta)}{20}} .
\end{aligned}
$$

This is used to obtain the necessary direction-dependent per-sample attenuation for each delay-line group using

$$
g_{i}(\phi, \theta)=\left(g_{\operatorname{lin}}(\phi, \theta)\right)^{m_{i}} .
$$

In practice, $T_{60}(\phi, \theta)$ is sampled uniformly in many directions, using a uniform set of points on a sphere, such as spherical $t$-designs of high degrees $(t \geq 20)$ [32].

\subsection{Determining the Recursive Transform}

The DWM is not applied in a single stage as is common in the reproduction of ambisonic signals $[35,36]$. Instead, it is included in the DFDN's recursion. For this purpose, $g_{i}(\phi, \theta)$ is used to construct a matrix operator that applies the desired gain in the $\mathrm{SH}$ domain. The $\mathrm{SH}$ coefficients of $g_{i}(\phi, \theta)$ are computed from Eq. (9) and the sampled values of $\boldsymbol{g}_{i}$ as

$$
\mathbf{g}_{i}=\frac{4 \pi}{K} \mathbf{Y}_{\mathrm{uni}}^{\mathrm{T}} g_{i}
$$

The coefficient vector $\mathbf{g}_{i}$ is an exact representation of the directional weighting in the SHD, instead of in the spatial domain.

Since the effective order $L^{\prime}$ of the directional weighting is unknown, we find the maximum $\mathrm{SH}$ order in $\mathbf{g}_{i}$ that contains $95 \%$ of the total energy of the coefficients. The coefficient vector $\mathbf{g}_{i}$ is then truncated up to the order $L^{\prime}$.

\subsection{Building the Matrix Operator}

Based on the final directional weighting expressed in the SHD $\mathbf{g}_{i}$ and its estimated order $L^{\prime}$, we construct the DWM $\mathbf{T}_{\text {full, }}$, which is illustrated in Fig. 4, realizing the recursive spatial shaping.

Starting from the output of the $i$ th delay line $s(n, \phi, \theta)$, the directional weighting $g(\phi, \theta)$, and their SHD representations $\mathbf{s}_{i}(n)$ and $\mathbf{g}_{i}$ of orders $L$ and $L^{\prime}$, respectively, we proceed as follows. The desired direction-dependent weighted signal $s^{\prime}(n, \phi, \theta)$ is given by

$$
s^{\prime}(n, \phi, \theta)=s(n, \phi, \theta) g_{i}(\phi, \theta) .
$$

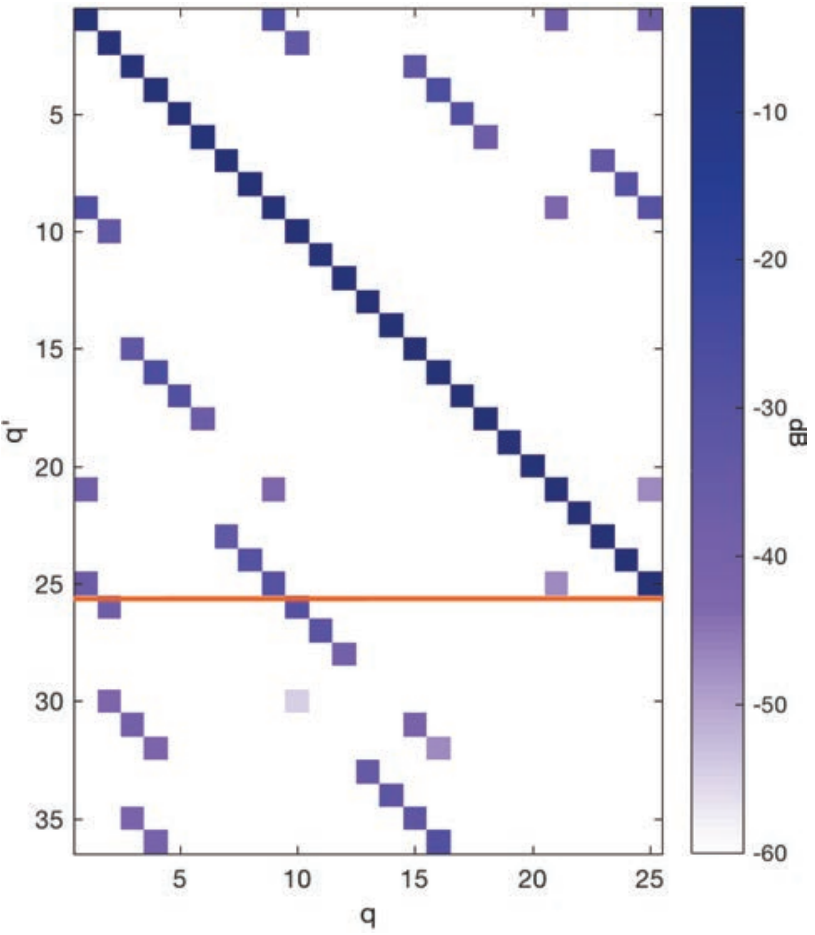

Fig. 4. The full transformation matrix of size $L$ by $L+L^{\prime}$. The values below the thick horizontal line are truncated in the final transform $\mathbf{T}_{i}$.

The DWM expresses the above equation directly in the SHD, having as its input the ambisonic signals $\mathbf{s}(n)$ and returning the modified ambisonic signals $\mathbf{s}^{\prime}(n)$ of order $L+L^{\prime}$

We use the following notation for compactness: a point on the unit sphere is $\mathbf{u} \equiv(\phi, \theta)$, and the spherical integration is denoted as $\int \mathrm{d} \mathbf{u}$. The vector $\mathbf{y}_{L}(\mathbf{u})$ denotes the $\mathrm{SH}$ vector of all SHs up to order $L$ in direction $\mathbf{u}$, and $Y_{q}(\mathbf{u})$ means the $q$ th $\mathrm{SH}$ in the series. Furthermore, we omit the sample index $n$ in the following expressions.

The directionally weighted ambisonic signals $\mathbf{s}^{\prime}$ express the SHT of the weighted sound scene

$$
\mathbf{s}^{\prime}=\operatorname{SHT}\left\{s^{\prime}(\mathbf{u})\right\}=\int g_{i}(\mathbf{u}) s(\mathbf{u}) \mathbf{y}_{L+L^{\prime}}(\mathbf{u}) \mathrm{d} \mathbf{u},
$$

where the $q$ th channel of $\mathbf{s}^{\prime}$ is

$$
s_{q}^{\prime}=\int g_{i}(\mathbf{u}) s(\mathbf{u}) Y_{q}(\mathbf{u}) \mathrm{d} \mathbf{u}
$$

Taking the inverse SHT, given in Eq. (10), of the product function and rearranging the terms, we obtain

$$
\begin{aligned}
s_{q}^{\prime} & =\operatorname{SHT}\left\{\operatorname{ISHT}\left\{\mathbf{g}_{i}\right\} \operatorname{ISHT}\{\mathbf{s}\}\right\} \\
& =\int\left(\mathbf{g}_{i}^{\mathrm{T}} \mathbf{y}_{L^{\prime}}(\mathbf{u})\right)\left(\mathbf{s}^{\mathrm{T}} \mathbf{y}_{L}(\mathbf{u})\right) Y_{q}(\mathbf{u}) \mathrm{d} \mathbf{u} \\
& =\mathbf{g}_{i}^{\mathrm{T}}\left(\int \mathbf{y}_{L^{\prime}}(\mathbf{u}) \mathbf{y}_{L}^{\mathrm{T}}(\mathbf{u}) Y_{q}(\mathbf{u}) \mathrm{d} \mathbf{u}\right) \mathbf{s} \\
& =\mathbf{g}_{i}^{\mathrm{T}} \mathbf{G}_{q} \mathbf{s},
\end{aligned}
$$


where $\mathbf{G}_{q}$ is a $(L+1)^{2} \times\left(L^{\prime}+1\right)^{2}$ matrix of Gaunt coefficients $G_{i j}^{q}$ for real spherical harmonics [39], given by

$$
\left[\mathbf{G}_{q}\right]_{i j}=G_{i j}^{q}=\int Y_{i}(\mathbf{u}) Y_{j}(\mathbf{u}) Y_{q}(\mathbf{u}) \mathrm{d} \mathbf{u} .
$$

Such coefficients can be computed analytically from Gaunt coefficients for complex SHs, for which closed-form solutions exist. Tabulated Gaunt coefficients for both complex and real SHs and orders up to $L+L^{\prime}=30$ are found in the accompanying code of [40].

The linear operation of Eq. (31) can be computed for all ambisonic signals $\mathbf{s}^{\prime}$ in one step:

$$
\mathbf{s}^{\prime}=\mathbf{T}_{\text {full }}\left(\mathbf{g}_{i}\right) \mathbf{s} .
$$

$\mathbf{T}_{\text {full }}$ is a $\left(L+L^{\prime}+1\right)^{2} \times(L+1)^{2}$ matrix with its $q$ th row given by

$$
\left[\mathbf{T}_{\text {full }}\left(\mathbf{g}_{i}\right)\right]_{q}=\mathbf{g}_{i}^{\mathrm{T}} \mathbf{G}^{q} .
$$

An alternative formulation to construct the matrix $\mathbf{T}_{\text {full }}\left(\mathbf{g}_{i}\right)$, given in [41], is to implement a discrete SHT form of Eq. (31) using a uniform spherical $t$-design with $t \geq 2\left(L+L^{\prime}\right)$ and then to evaluate the weighting function for that set of points:

$$
\mathbf{T}_{\text {full }}\left(\mathbf{g}_{i}\right)=\frac{4 \pi}{K} \mathbf{Y}_{L+L^{\prime}}^{\mathrm{T}} \operatorname{diag}\left[\mathbf{Y}_{L^{\prime}} \mathbf{g}_{i}\right] \mathbf{Y}_{L}
$$

where $\mathbf{Y}_{L+L^{\prime}}$ is the matrix of SHs up to order $L+L^{\prime}$ computed in the $K$ directions of the $t$-design and the $\operatorname{diag}[\cdot]$ operator constructs a diagonal matrix from a vector.

\subsection{Ambisonic Order Preservation}

One issue when integrating the directional modification into the recursion is that increasing the order of the output ambisonic channels by as much as the order $L^{\prime}$ of the directional weighting becomes impractical. A practical solution is to use an approximation of the full transformation matrix $\mathbf{T}_{\text {full }}\left(\mathbf{g}_{i}\right)$ that is square, hence preserving the order and the number of channels. We select a truncated $\mathbf{T}_{i}$ that retains only the first $(L+1)^{2}$ rows of $\mathbf{T}_{\text {full }}\left(\mathbf{g}_{i}\right)$ (see Fig. 4):

$$
\mathbf{T}_{i}=\mathbf{T}_{\text {full }}\left(\mathbf{g}_{i}\right)\left[1,2, \ldots(L+1)^{2} ; 1,2, \ldots(L+1)^{2}\right] .
$$

Due to the truncation, the direction-dependent decay times do not match exactly the target ones. However, since the application considered here is late directional reverberation design, exact matching is not necessary.

We demonstrate the effect of the truncated recursive transform matrix in the results section through comparisons with a simulated room. A demonstration of the effect is given in Fig. 5, where an example transform is applied recursively to an omnidirectional signal and the resulting signal is visualized at different time steps.

\section{RESULTS}

A case study is presented here to evaluate the directiondependent energy decay characteristics of the DFDN reverberator and to compare it with a simulated acoustic space. For this purpose, we implemented an image-source algorithm [42] to serve as an ideal representation of the

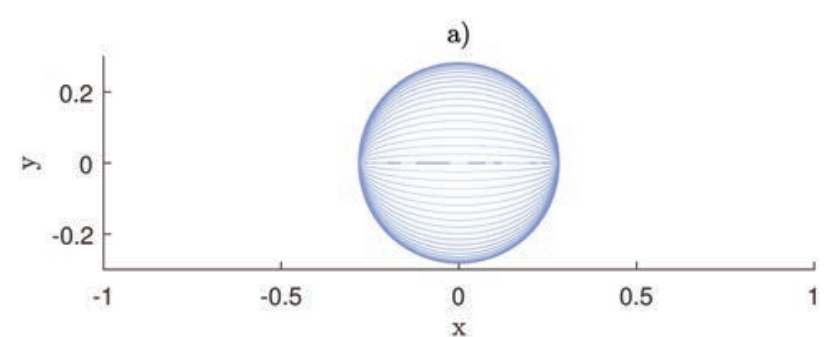

b)

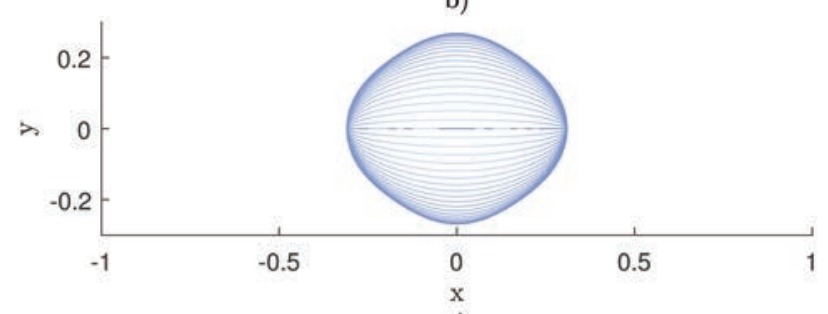

c)

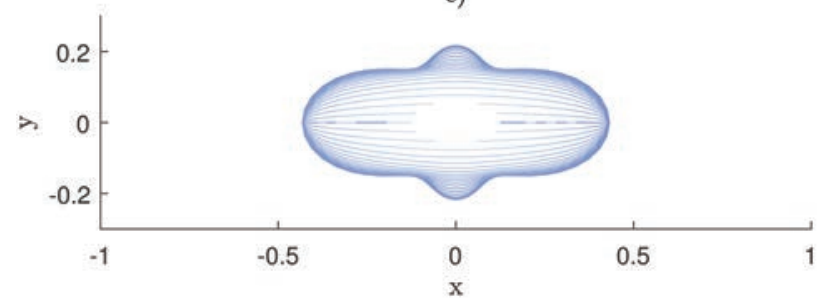

Fig. 5. Direction-dependent energy (Eq. (28), expressed on a linear scale of the Cartesian coordinate system, at different recursive steps (a) before applying the transform, (b) after half of the target iterations (50/100), and (c) after the target number of iterations (100).

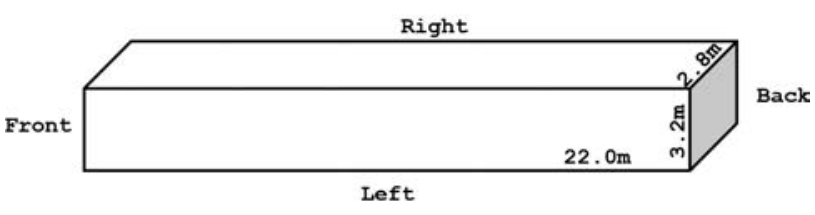

Fig. 6. Layout of the simulated room.

direction-dependent energy distribution over time. We then designed a DWM for the DFDN that approximates a similar distribution to the one obtained with the image-source method. We studied a shoebox type of room having a distinctively long shape for this purpose. This special case is equivalent to a long corridor that clearly exhibits anisotropic energy decay.

\subsection{Image-Source Reference}

Using the image-source method, we set the dimensions of a rectangular room to be $22 \mathrm{~m} \times 2.8 \mathrm{~m} \times 3.2 \mathrm{~m}$, as illustrated in Fig. 6, and place both the source and listener close to the center of the room. All generated reflections were encoded as a broadband impulse using their simulated directions of arrival to encode them into an SIR. This SIR was then analyzed and used to inform the design of the DFDN. 
(a)

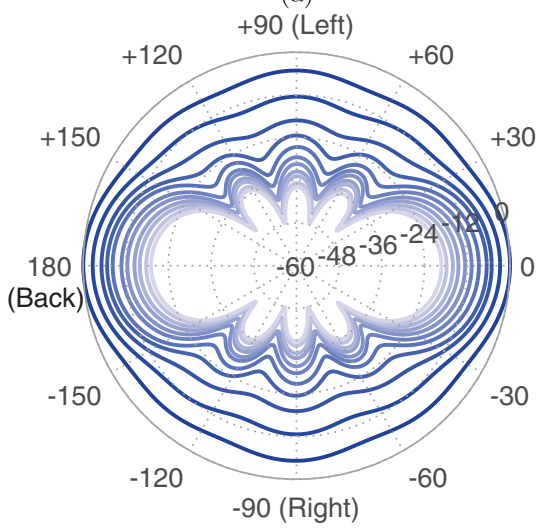

(b)

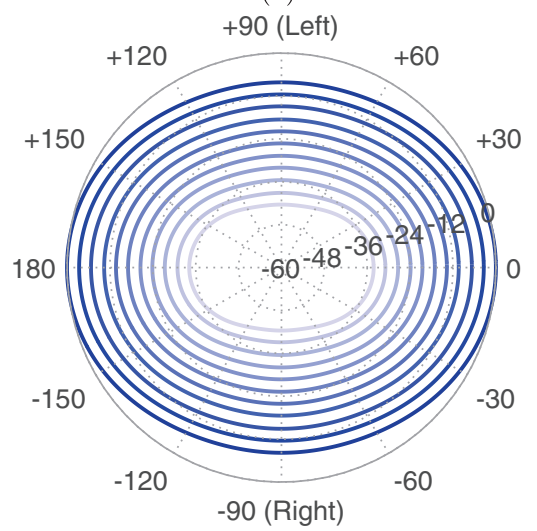

(c) +90 (Left)

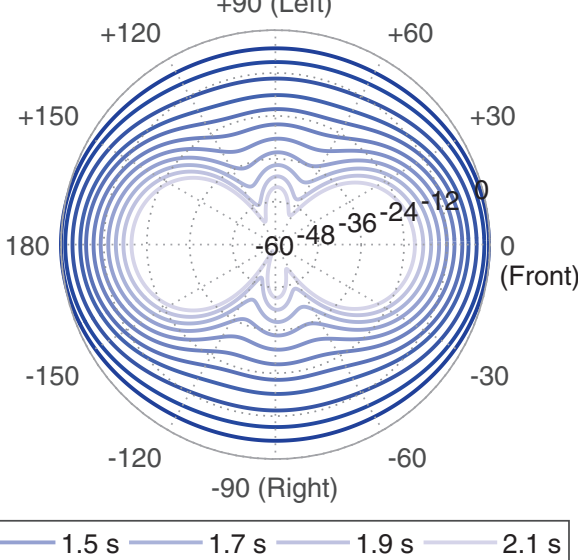

Fig. 7. Directional energy distribution, in $\mathrm{dB}$, for consecutive time segments using (a) the image-source method, (b) the conventional FDN with single-channel delay lines and a weighted directional output, and (c) the DFDN designed to resemble the SIR of the image-source method.

\subsection{Design Consideration}

The DFDN has been designed as an extension of a fourdelay-line FDN using third-order Ambisonics, which requires 16 channels to achieve a good level of directionality. Therefore, the reverberator has 4 groups of 16 delay lines, making a total of 64 delay lines. A gain factor was applied for broadband attenuation, but no frequency-dependent filters, for simplicity.

The $g_{i}(\phi, \theta)$ used for the DWM design are calculated from $T_{60}(\phi, \theta)$ values, which are obtained by extracting directional impulse responses (DIR) through beamforming from the output of the image source. The beamforming analysis is performed in the SHD using a plane-wave decomposition method based on Eq. (11). The grid used to select the beamforming sampling locations is a spherical $t$-design suitable for the SHT up to order 20 without error. Each $\mathbf{T}_{i}$ is obtained from the per-sample transform calculated in Eq. (26) and the individual randomly selected delay length $m_{i}$.

\subsection{Directional Analysis}

To analyze the energy distribution of the generated DIR, we again sample the ambisonic signals in different directions and time windows through a beamforming analysis. The horizontal plane at $0^{\circ}$ elevation is sampled at $1^{\circ}$ intervals which yields a 2-dimensional, direction-dependent energy distribution corresponding to a view from above the plane. In Fig. 7(a), we visualize the time and directiondependent energy distribution of the room simulated using the image-source method. In the initial time window (the outermost curve in Fig. 7(a)) shows that the energy distribution behaves nearly isotropically. However, an approximately $6-\mathrm{dB}$ difference is already present between the right-left and the front-back axis. Shortly into the simulation, the directionality of the reflections converges towards the long end of the corridor (front, back) with an amplitude deviation in the order of $20 \mathrm{~dB}$ of the last segment. Therefore, the energy decay is anisotropic in this case.
Fig. 7(b) shows the results obtained with a conventional FDN using single-channel delay lines, designed for references purposes. A directional gain is applied to the output before being encoded it into Ambisonics, similarly to the method presented in [26]. The desired output is obtained after computing the impulse response by sending a single impulse through the system. We observe from the segmented output that the energy decay time remains constant in all direction since the gain factor is only applied to the final output of the reverberator. Although the sound field is anisotropic, for this example, the energy decay itself is considered isotropic, since the oval shape of the curve is preserved at all time instances.

In Fig. 7(c), an impulse is sent through the DFDN to generate a DIR. We see that the first segments begin in an isotropic energy distribution that then converges towards a direction-dependent energy distribution. Here, the DFDN demonstrates an anisotropic energy decay, similar to the one observed in Fig. 7(a).

\subsection{Computational Cost Considerations}

When comparing the computational costs to a conventional FDN, the DFDN's costs increases by factor equivalent to the number of channels in the delay-line groups. In our test case, third-order Ambisonics delay-line groups were used to ensure a detailed directional analysis. However, since third-order Ambisonics requires 16 channels, this increases the number of delay lines by a factor of 16 when compared to an equivalent conventional FDN. Since FDNs are often designed in the vicinity of 32 or even 64 delay lines to ensure a sufficiently high modal and echo density, a factor of 16 is relatively significant. IIR filtering in the recirculating signal path for frequency-dependent decay is another a common design feature that increases the computational costs of each delay line. Therefore, selecting the appropriate Ambisonics order for practical use is important to limit the computational cost and will vary depending on the desired spatial accuracy of the direction-dependent 
reverberator. However, since the perceptual attributes of anisotropic energy decay have yet to be fully established, the optimal Ambisonics order should be chosen based on the intended application and an empirical evaluation.

\section{CONCLUSION}

This paper presented a novel method to extend the FDN reverberator design to render anisotropic energy decay characteristics. By expanding delay lines into delay-line groups, or multichannel delay lines, containing individual ambisonic channels, we maintain the direction-dependent energy distribution within the recirculating system. Through a matrix transform, which modifies the direction-dependent energy distribution in the $\mathrm{SH}$ domain, we alter this distribution and approximate the direction-dependent energy decay of an existing DIR. Since all of the properties of the original FDN are preserved, the proposed DFDN is built by extending an existing FDN configuration with the multichannel elements of the DFDN. Further research is needed to fully establish the appropriate spatial definition required to achieve a good compromise between the perception of direction-dependent decay and computational costs.

\section{ACKNOWLEDGMENT}

This work has been funded in part by the Academy of Finland (ICHO project, Aalto University project No. 13296390) and has been related to the activities of the Nordic Sound and Music Computing NetworkNordicSMC (NordForsk project no. 86892).

\section{REFERENCES}

[1] M. R. Schroeder and B. F. Logan, "'Colorless' Artificial Reverberations," J. Audio Eng. Soc., vol. 9, no. 3, pp. 192-197 (1961 Jul.).

[2] V. Välimäki, J. D. Parker, L. Savioja, J. O. Smith, and J. S. Abel, "Fifty Years of Artificial Reverberation," IEEE Trans. Audio Speech Lang. Process., vol. 20, no. 5, pp. 1421-1448 (2012 Jul.).

[3] S. J. Schlecht and E. A. P. Habets, "Feedback Delay Networks: Echo Density and Mixing Time," IEEE/ACM Trans. Audio Speech Lang. Process., vol. 25, no. 2, pp. 374-383 (2017 Feb.).

[4] J.-M. Jot and A. Chaigne, "Digital Delay Networks for Designing Artificial Reverberators," presented at the 90th Convention of the Audio Engineering Society (1991 Feb.), convention paper 3030.

[5] S. J. Schlecht and E. A. Habets, "Accurate Reverberation Time Control in Feedback Delay Networks," in Proceedings of the International Conference on Digital Audio Effects (DAFx-17), pp. 337-344 (Edinburgh, UK) (2017 Sep.).

[6] M. A. Gerzon, "Synthetic Stereo Reverberation: Part I," Studio Sound, vol. 13, pp. 632-635 (1971 Dec.).

[7] M. A. Gerzon, "Synthetic Stereo Reverberation: Part II,” Studio Sound, vol. 14, pp. 24-28 (1972 Jan.).
[8] J. Stautner and M. Puckette, "Designing MultiChannel Reverberators," Computer Music J., vol. 6, no. 1, pp. 52-65 (1982 Spring).

[9] D. Rocchesso and J. O. Smith, "Circulant and Elliptic Feedback Delay Networks for Artificial Reverberation," IEEE Trans. Speech Audio Process., vol. 5, no. 1, pp. 5163 (1997 Jan.).

[10] S. J. Schlecht, E. A. P. Habets, “On Lossless Feedback Delay Networks," IEEE Trans. Signal Process., vol. 65, no. 6, pp. 1554-1564 (2017 Mar.).

[11] E. D. Sena, H. Hacıhabiboğlu, Z. Cvetković, and J. O. Smith, "Efficient Synthesis of Room Acoustics via Scattering Delay Networks," IEEE/ACM Trans. Audio Speech Lang. Process., vol. 23, no. 9, pp. 1478-1492 (2015 Sep.).

[12] F. Stevens, D. T. Murphy, L. Savioja, and V. Välimäki, "Modeling Sparsely Reflecting Outdoor Acoustic Scenes Using the Waveguide Web," IEEE/ACM Trans. Audio Speech Lang. Process., vol. 25, no. 8, pp. 1566-1578 (2017 Aug.).

[13] S. J. Schlecht, and E. A. P. Habets, "Sign-Agnostic Matrix Design for Spatial Artificial Reverberation with Feedback Delay Networks," presented at the Audio Engineering Society International Conference on Spatial Reproduction-Aesthetics and Science (2018 Aug.), conference paper P12-1.

[14] V. Pulkki, "Spatial Sound Reproduction with Directional Audio Coding," J. Audio Eng. Soc., vol. 55, no. 6, pp. 503-516 (2007 Jun.).

[15] A. D. Pierce, "Concept of a Directional Spectral Energy Density in Room Acoustics," J. Acoust. Soc. Am., vol. 56, no. 4, pp. 1304-1305 (1974 Oct.).

[16] C. G. Balachandran and D. W. Robinson, "Diffusion of the Decaying Sound Field," Acta Acust., vol. 19, no. 5, pp. 245-257 (1967 Jan.).

[17] D. Lubman, "Traversing Microphone Spectroscopy as a Means for Assessing Diffusion," J. Acoust. Soc. Am., vol. 56, no. 4, pp. 1302-1304 (1974 Oct.).

[18] B. N. Gover, J. G. Ryan, and M. R. Stinson, "Microphone Array Measurement System for Analysis of Directional and Spatial Variations of Sound Fields," J. Acoust. Soc. Am., vol. 112, no. 5, pp. 1980-1991 (2002 Nov.).

[19] M. Berzborn and M. Vorländer, "Investigations on the Directional Energy Decay Curves in Reverberation Rooms," in Proceedings of Euronoise (Crete, Greece) (2018 May).

[20] D. Romblom, C. Guastavino, and P. Depalle, "Perceptual Thresholds for Non-Ideal Diffuse Field Reverberation," J. Acoust. Soc. Am., vol. 140, no. 5, pp. 3908-3916 (2016 Nov.).

[21] M. A. Gerzon, "Ambisonics in Multichannel Broadcasting and Video," J. Audio Eng. Soc., vol. 33, no. 11, pp. 859-871 (1985 Nov.).

[22] M. A. Gerzon, "Recording Concert Hall Acoustics for Posterity," J. Audio Eng. Soc., vol. 23, no. 7, pp. 569571 (1975 Sep.).

[23] J. Merimaa and V. Pulkki, "Spatial Impulse Response Rendering I: Analysis and Synthesis," J. Audio Eng. Soc., vol. 53, no. 12, pp. 1115-1127 (2005 Dec.). 
[24] S. Oksanen, J. Parker, A. Politis, and V. Välimäki, "A Directional Diffuse Reverberation Model for Excavated Tunnels in Rock," in Proceedings of the IEEE International Conference on Acoustics, Speech and Signal Processing (ICASSP), pp. 644-648 (Vancouver, Canada) (2013 May).

[25] L. Savioja and U. P. Svensson, "Overview of Geometrical Room Acoustic Modeling Techniques," J. Acoust. Soc. Am., vol. 138, no. 2, pp. 708-730 (2015 Aug.).

[26] J. Anderson and S. Costello, "Adapting Artificial Reverberation Architectures for B-Format Signal Processing," in Proceedings of the Ambisonics Symposium, Graz, Austria (2009 Jun.).

[27] B. Wiggins and M. Dring, "AmbiFreeVerb 2Development of a 3D Ambisonic Reverb with Spatial Warping and Variable Scattering," presented at the Audio Engineering Society International Conference on Sound Field Control (2016 Jul.), conference paper 2-3.

[28] M. R. Schroeder, "New Method of Measuring Reverberation Time," J. Acoust. Soc. Am., vol. 37, no. 3, pp. 409-412 (1965 Mar.).

[29] D. G. Malham and A. Myatt, "3-D Sound Spatialization Using Ambisonic Techniques," Computer Music J., vol. 19, no. 4, pp. 58-70 (1995 Winter).

[30] F. Zotter, Analysis and Synthesis of SoundRadiation With Spherical Arrays, Ph.D. thesis, Institute of Electronic Music and Acoustics, University of Music and Performing Arts, Graz, Austria (2009 Sep.).

[31] A. Politis and D. Poirier-Quinot, "JSAmbisonics: A Web Audio Library for Interactive Spatial Sound Processing on the Web," presented at the Interactive Audio Systems Symposium (2016 Sep.).

[32] R. H. Hardin and N. J. Sloane, "McLarens Improved Snub Cube and Other New Spherical Designs in Three Dimensions," Discrete Comput. Geom., vol. 15, no. 4, pp. 429-441 (1996 Jul.).

[33] M. A. Gerzon, and G. J. Barton, "Ambisonic Decoders for HDTV," presented at the 92nd Convention of the
Audio Engineering Society (1992 Mar.), convention paper 3345.

[34] F. Zotter and H. Pomberger, "Warping of the Recording Angle in Ambisonics," in Proceedings of the First International Conference on Spatial Audio (ICSA) (Detmold, Germany) (2011 Jan.).

[35] M. Kronlachner and F. Zotter, "Spatial Transformations for the Enhancement of Ambisonic Recordings," in Proceedings of the Second International Conference on Spatial Audio (Erlangen, Germany) (2014 Feb.).

[36] P. Lecomte, P.-A. Gauthier, A. Berry, A. Garcia, and C. Langrenne, "Directional Filtering of Ambisonic Sound Scenes," presented at the AES International Conference on Spatial Reproduction-Aesthetics and Science (2018 Aug.), conference paper P7-1.

[37] P. A. Regalia and S. K. Mitra, "Kronecker Products, Unitary Matrices and Signal Processing Applications," SIAM Review, vol. 31, no. 4, pp. 586-613 (1989 Dec.).

[38] H. Zhang and F. Ding, "On the Kronecker Products and Their Applications," J. Appl. Math., vol. 2013, pp. 296185:1-296185:8 (2013 Jun.).

[39] H. H. Homeier and E. O. Steinborn, "Some Properties of the Coupling Coefficients of Real Spherical Harmonics and Their Relation to Gaunt Coefficients," J. Mol. Struct.: THEOCHEM, vol. 368, pp. 31-37 (1996 Sep.).

[40] A. Politis, Microphone Array Processing for Parametric Spatial Audio Techniques, Ph.D. thesis, Aalto University, Espoo, Finland (2016 Oct.).

[41] M. Kronlachner and F. Zotter, "Spatial Transformations for the Enhancement of Ambisonic Recordings," in Proceedings of the Second International Conference on Spatial Audio (ICSA) (Erlangen, Germany) (2014 Feb.).

[42] J. B. Allen and D. A. Berkley, "Image Method for Efficiently Simulating Small-Room Acoustics," J. Acoust. Soc. Am., vol. 65, no. 4, pp. 943-950 (1979 Apr.). 
THE AUTHORS

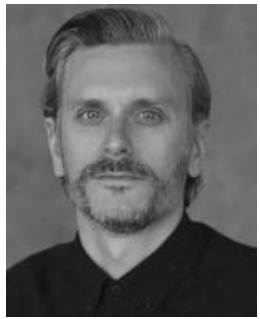

Benoit Alary

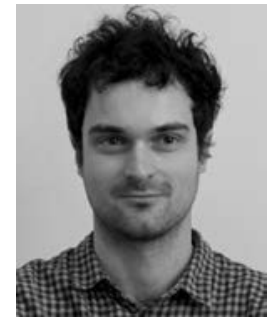

Archontis Politis

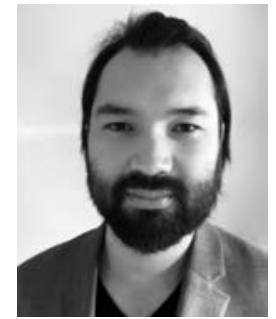

Sebastian J. Schlecht

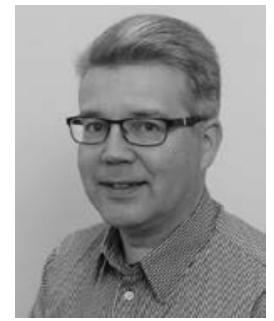

Vesa Välimäki

Benoit Alary received a B.Sc. degree in Computer Science from the Université de Montréal, Canada, in 2005 and a M.Sc. degree in Acoustics and Music Technologies from the University of Edinburgh in 2011. He is currently a doctoral candidate in the Acoustics Lab of Aalto University in Espoo, Finland, where he is researching immersive reverberation algorithms. Along with his academic work, he has over 10 years of experience developing interactive audio technologies for the video game industry, where he specialized in the development of virtual acoustics systems. His research interests include reverberation algorithms, virtual acoustics, spatial audio, and decorrelation techniques.

Archontis Politis obtained his M.Eng. degree in civil engineering from Aristotle University, Thessaloniki, Greece, and his M.Sc. degree in Sound \& Vibration studies from the Institute of Sound and Vibration Research (ISVR), Southampton, UK, in 2006 and 2008, respectively. From 2008 to 2010 he worked as a graduate acoustic consultant in Arup Acoustics, UK, and as a researcher in a joint collaboration between Arup Acoustics and the Glasgow School of Arts on architectural auralization using spatial sound techniques. In 2016 he obtained a Doctor of Science degree on parametric spatial sound recording and reproduction from Aalto University, Finland. He has also completed an internship at the Audio and Acoustics Research Group of Microsoft Research during the summer of 2015. He received an AES Convention best student paper award in 2013. He has held workshops on parametric spatial audio processing, and he has co-authored a book on the topic, published in 2017 . He is currently a post-doctoral research fellow at the Audio and Signal Processing Research Group, Tampere University, Finland. His research interests include spatial audio technologies, virtual acoustics, array signal processing, and acoustic scene analysis.

Sebastian J. Schlecht is a Professor of Practice for Sound in Virtual Reality at Aalto University, Espoo, Finland. He received the Diploma in Applied Mathematics from the University of Trier, Germany, in 2010 and the M.Sc. degree in Digital Music Processing from School of Electronic Engineering and Computer Science at Queen Mary University of London, UK, in 2011. In 2017, he received a doctoral degree at the International Audio Laboratories Erlangen,

Germany, on artificial spatial reverberation and reverberation enhancement systems. From 2012 on, Dr. Schlecht was also an external researcher and the lead developer of the 3D Reverb algorithm at the Fraunhofer IIS, Erlangen, Germany. In 2018, he received with B. Alary, V. Välimäki, and E.A.P. Habets the Best Paper Award at the International Conference on Digital Audio Effects in Aveiro, Portugal. In the same year, he was the recipient, with O. Rummukainen, T. Robotham, A. Plinge, J. Herre, and E.A.P. Habets of the Best Peer-Reviewed Paper at the AES International Conference on Audio for Virtual and Augmented Reality in Redmond, WA, USA. His research interests are acoustic modeling and auditory perception of acoustics, analysis, and synthesis of feedback systems, music information retrieval, and virtual and augmented reality. $\mathrm{He}$ is also an active live and studio musician in contemporary and popular music.

Vesa Välimäki is Professor of Audio Signal Processing at Aalto University, Espoo, Finland. He is the Vice Dean for Research in the Aalto University School of Electrical Engineering. He received his M.Sc. in Technology and Doctor of Science in Technology degrees in electrical engineering from the Helsinki University of Technology (TKK) in 1992 and 1995, respectively. His doctoral dissertation dealt with fractional delay filters and physical modeling of musical instruments. In 1996, he was a Postdoctoral Research Fellow at the University of Westminster, London, UK. In 20012002, he was Professor of Signal Processing at the Pori School of Technology and Economics, Tampere University of Technology, Pori, Finland. In 2006-2007, he was the head of the TKK Laboratory of Acoustics and Audio Signal Processing. During the academic year 2008-2009, he was a visiting scholar at the Center for Computer Research in Music and Acoustics (CCRMA), Stanford University, Stanford, CA, USA. His research interests include digital filter design, loudspeaker and headphone signal processing, audio effects processing, and sound synthesis. Prof. Välimäki is a Fellow of the AES, a Fellow of the IEEE, and a Life Member of the Acoustical Society of Finland. He was the General Chair of the 11th International Conference on Digital Audio Effects DAFx-08 in 2008, and the General Chair of the 14th International Sound and Music Computing Conference SMC-17 in 2017. 